\title{
Studying solutions at high shear rates: a dedicated microfluidics setup
}

\author{
D. C. F. Wieland, V. M. Garamus, T. Zander, C. Krywka, M. Wang, A. \\ Dedinaite, P. M. Claesson and R. Willumeit-Römer
}

J. Synchrotron Rad. (2016). 23, 480-486

\section{IUCr Journals CRYSTALLOGRAPHY JOURNALS ONLINE}

Copyright (C) International Union of Crystallography

Author(s) of this paper may load this reprint on their own web site or institutional repository provided that this cover page is retained. Republication of this article or its storage in electronic databases other than as specified above is not permitted without prior permission in writing from the IUCr.

For further information see http://journals.iucr.org/services/authorrights.html 


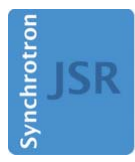

JOURNAL OF SYNCHROTRON RADIATION

ISSN 1600-5775

Received 16 October 2015

Accepted 28 December 2015

Edited by S. Svensson, Uppsala University, Sweden

Keywords: shear; complex solutions; small-angle X-ray scattering (SAXS); microfluidics; lysozyme; aggregation; rheology; protein interaction; beamline setup.

Supporting information: this article has supporting information at journals.iucr.org/s

\section{Studying solutions at high shear rates: a dedicated microfluidics setup}

\author{
D. C. F. Wieland, ${ }^{a} *$ V. M. Garamus, ${ }^{a}$ T. Zander, ${ }^{a}$ C. Krywka, ${ }^{a}$ M. Wang, ${ }^{b, c}$ \\ A. Dedinaite, $^{b, c}$ P. M. Claesson ${ }^{b, c}$ and R. Willumeit-Römer ${ }^{a}$
}

anstitute for Materials Research, Helmholtz-Zentrum Geestacht: Centre for Materials and Coast Research, Max-Planck-Strasse 1, Geesthacht 21502, Germany, ${ }^{\mathbf{b}}$ Department of Chemistry, Surface and Corrosion Science, KTH Royal Institute of Technology, Drottning Kristinas väg 51, Stockholm 10044, Sweden, and ' SP Chemistry, Materials and Surfaces, SP Technical Research Institute of Sweden, Box 5607, Stockholm SE-114 86, Sweden. *Correspondence e-mail: florian.wieland@embl-hamburg.de

The development of a dedicated small-angle X-ray scattering setup for the investigation of complex fluids at different controlled shear conditions is reported. The setup utilizes a microfluidics chip with a narrowing channel. As a consequence, a shear gradient is generated within the channel and the effect of shear rate on structure and interactions is mapped spatially. In a first experiment small-angle X-ray scattering is utilized to investigate highly concentrated protein solutions up to a shear rate of $300000 \mathrm{~s}^{-1}$. These data demonstrate that equilibrium clusters of lysozyme are destabilized at high shear rates.

\section{Introduction}

The rheological response of complex fluids is to a great extent determined by their supramolecular structure. In order to gain insight and to understand rheological responses in more detail it is necessary to combine rheometry with other experimental methods that allow monitoring of structural changes induced by shear forces. X-ray scattering techniques open the possibility to study the structure of different material classes in situ and also time-resolved. Therefore, these techniques have been applied to study stable and flocculated colloidal suspensions (Pignon et al., 1997; Jogun \& Zukoski, 1999; Versmold et al., 2001; Panine et al., 2002; Hoekstra et al., 2005), surfactant systems (Diat et al., 1995; Roux et al., 1995; Molino et al., 1998; With et al., 2014), liquid crystalline systems (Hongladarom et al., 1996; Burghardt, 1998; Hoekstra et al., 2002; Cinader \& Burghardt, 1999), flow-enhanced crystallization in polymers (Kumaraswamy et al., 1999; Agarwal et al., 2003; Somani et al., 2000; Liu, Zhou et al., 2011) and polymer nanocomposites (Pujari et al., 2011; Dykes et al., 2012). The advance of highly brilliant X-ray beams in combination with intense X-ray sources enables small-angle X-ray scattering (SAXS) techniques to access a wide range of length scales $\left(q=0.001 \mathrm{~nm}^{-1}\right.$ $5 \mathrm{~nm}^{-1}$ ) with a high resolution during flow of liquids and, also, good time resolution (Hoekstra et al., 2005). SAXS has therefore become a well suited tool for studying the origins of non-linear response effects of microstructures undergoing structural changes. Small-angle X-ray scattering can provide insight into the effects of shear on the shape, orientation and size of individual objects (Trebbin et al., 2013). Changes of individual objects can be characterized by their form factor, whereas changes in the spatial organization can be investigated through the structure factor (Evans et al., 2007). 
The existing rheometry setups for scattering experiments are quite complex. We have designed an alternative that uses a microfluidics chip. The advantages of this setup over conventional rheometers are the high flexibility and versatility of the microfluidics device that can easily be used at different beamlines. The setup has low weight and overall small dimensions and thus puts less constrains on the available space and motor stages. It utilizes a shear gradient that is generated along the channel of the microfluidics chip. With this concept the effect of different shear rates on structure and interactions can be accessed by probing different positions in the channel. Very high shear rates can be achieved, which is not easy to reach with conventional rheometers.

In a first set of experiments we investigated the behaviour of a colloidal system to explore the versatility and functionality of the microfluidics device. The structure and dynamics of colloidal systems, having short-range attractive and long-range screened Coulombic repulsive interactions, have been extensively investigated in recent years. This type of interaction is ubiquitous and can be found in many charged colloid systems such as sols of proteins or poly(methyl methacrylate) (PMMA) particles with a polymer-introduced depletion attraction (Chen et al., 2007; Liu et al., 2005; Groenewold \& Kegel, 2001; Sciortino et al., 2004, 2005; Toledano et al., 2009; Shukla et al., 2008; Stradner et al., 2004, 2006; Lonetti et al., 2004; Campbell et al., 2005; Wu et al., 2004).

Solutions of proteins show an interesting feature in this context as the proteins may form equilibrium clusters. These clusters were noticed early in a cytochrome $c$ protein gel using small-angle neutron scattering (SANS) experiments, where a diffraction peak at low $q$ was observed (Lonetti et al., 2004). Such a peak has also been found in lysozyme solutions. It was called 'cluster-peak', and located at smaller scattering angles than the monomer-monomer interaction peak (Stradner et al., 2004) and, as the name implies, assigned to the formation of finite lysozyme clusters (Liu et al., 2005; Stradner et al., 2004, 2006; Cardinaux et al., 2011; Broccio et al., 2006). Different theoretical investigations exploring equilibrium structures in aqueous lysozyme solutions indeed suggest the existence of such clusters (Campbell et al., 2005; Kowalczyk et al., 2011; Sciortino et al., 2005; Toledano et al., 2009). It is not straightforward to apply the knowledge gained from a structurally homogeneous colloidal system, e.g. PMMA sols, directly to protein clusters as the proteins exhibit charged patches, heterogenic distribution of hydrophobic and hydrophilic patches, and shape irregularities. Nevertheless, lysozyme is normally treated as a simple colloidal with a spherical shape and an isotropic potential. This approximation is an oversimplification, but it is able to catch most of the physical features of lysozyme solutions. For example, adding excessive salt to a lysozyme solution transforms the solubility diagram of lysozyme to a typical phase diagram of colloids which only shows short-range attractions (Rosenbaum et al., 1996). Therefore, it is generally accepted that the interactions of lysozyme in solution can be explained by short-range attraction and long-range screened Coulomb repulsion (Chen et al., 2007; Tardieu et al., 1999).
On the other hand, a considerable number of investigations put doubt on the existence of equilibrium clusters in lysozyme solutions (Shukla et al., 2008). For instance, it has been suggested that the data could also be fitted by applying a double Yukawa potential model (Chen et al., 2007; Liu et al., 2005; Broccio et al., 2006). A further study concluded that the data could be interpreted by a model that predicts an intermediate-range structure rather than by a correlation of clusters (Liu, Porcar et al., 2011). However, still other studies employing SANS and NSE (neutron spin echo) stated that dynamic lysozyme clusters form with a finite lifetime in equilibrium with monomers (Porcar et al., 2010; Kowalczyk et al., 2011).

The equilibrium state in aqueous solutions of lysozyme is likely influenced by external parameters (temperature, pressure, shear, ...) as well as exact solution composition. Here, we aim to investigate how lysozyme solutions behave under shear, and how the interaction changes in the presence of shear forces. To this end we have performed SAXS experiments on $50 \mathrm{mg} \mathrm{ml}^{-1}$ (volume fraction $\varphi=0.034$ ) lysozyme solutions of two ionic strengths at $\mathrm{pH} 7$ to vary the screening of long-range electrostatic interactions.

\section{Microfluidic design and setup}

We have constructed a miniaturized rheology setup based on a microfluidics chip in which the effects of different shear rates are mapped spatially along a narrowing channel. Thus, solutions exposed to different shear rates are probed by making a two-dimensional scan across the channel. In order to generate such a shear gradient in a microfluidics chip we used a design where the width of the channel decreases along the flow direction of the liquid. Figs. 1( $a$ ) and 1(b) show CAD drawings of the microfluidics chip. The channel has an initial width of $100 \mu \mathrm{m}$, which decreases to $10 \mu \mathrm{m}$ over a path length of $100 \mu \mathrm{m}$. The narrowing in the channel had a hyperbolic shape, as it was observed to be preferential for the fabrication of micro-rheometers (Oliveira et al., 2007). The corner of the channel on the left-hand side that was located $300 \mu \mathrm{m}$ away from the beginning of the narrowing, see Fig. 1(a), was used as a reference point. This enabled a precise positioning of the microfluidics chip. The chips, with outer dimension $10 \mathrm{~mm} \times$ $20 \mathrm{~mm}$, were produced by MicroLiquid (Spain) using lithography techniques. The depth of the channels (from the top lid) is $200 \mu \mathrm{m}$ resulting in an aspect ratio of 20 at the narrowest part of the channel where the channel height is $10 \mu \mathrm{m}$. The chips were fabricated using an epoxy-based photoresist, SU8, which is a standard material used for lithography. The cover and bottom lid had thicknesses of $50 \mu \mathrm{m}$ and were also made of SU8. The windows material has a contribution in the smallangle scattering regime, which can be subtracted from the obtained scattering patterns. The absorbance of the windows is less than $1 \%$. Thus, the total thickness of the microfluidics chip was $300 \mu \mathrm{m}$. The chips were designed to be one-time-use only. During the experiment the chip was placed in a dedicated holder that provided the connection to the tubing system. This holder consisted of two plates with a rectangular opening of 


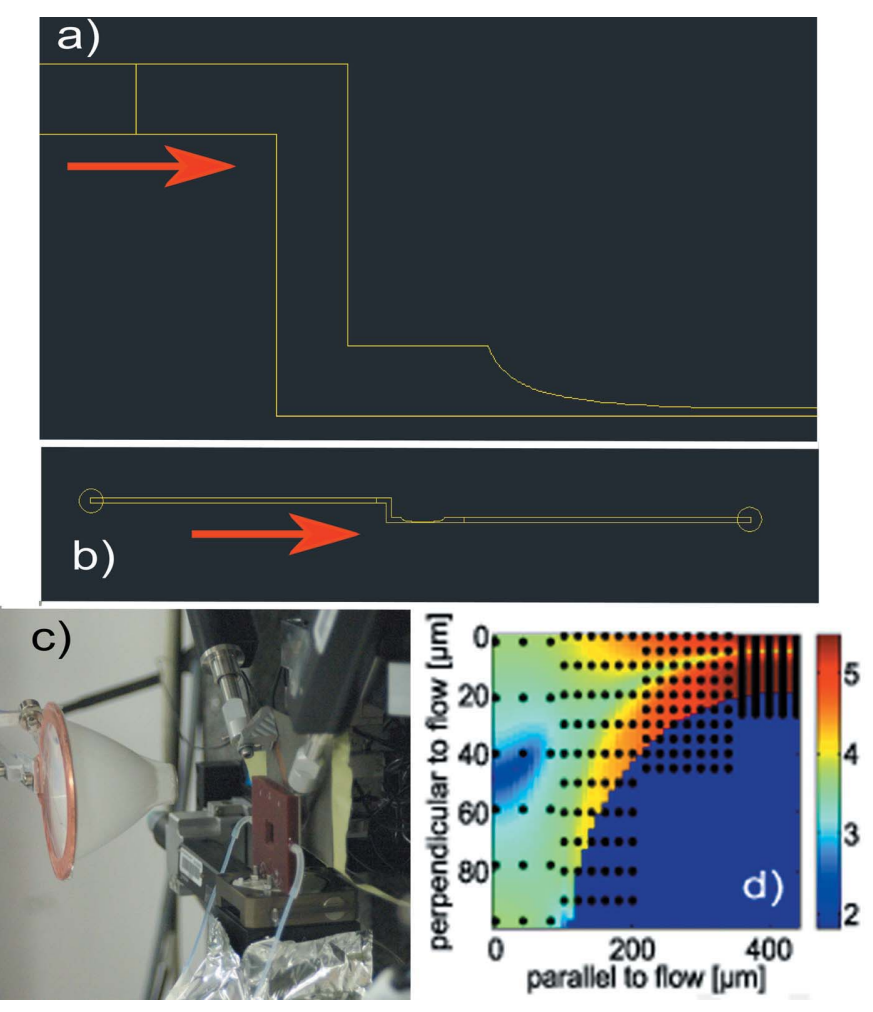

Figure 1

Photographs and drawings of the microfluidics setup. (a) Enlarged CAD drawing of the microfluidics chip showing the narrowing of the chip. The red arrow indicates the liquid flow direction. (b) CAD drawing of the microfluidics chip. (c) Microfluidics chip installed at ID13. (d) Twodimensional map of the shear rate distribution on the microfluidics channel. The colour bar represents the logarithmic shear rate. The black points denote the mesh scan made across the channel.

$16 \mathrm{~mm} \times 8 \mathrm{~mm}$ to perform transmission experiments, allowing scattering angles up to $80^{\circ}$ to be accessed. The chip was clamped between these two plates and secured by six screws. Holes were drilled in one of the plates, which served as piping for the fluid. O-rings were placed between this plate and the inlet/outlet of the microfluidics chip to ensure a tight sealing. A pulsation-free syringe pump, type neMESYS from Cetoni, Germany, was used with a low 14:1 gear to pump the liquid, see Fig. 1(c). The size of the syringe was chosen depending on the volume pump rate. For the measurements described in this paper, syringes with a volume of $2 \mathrm{ml}$ were used. The whole setup is compatible with flow rates of $10 \mu \mathrm{l} \mathrm{s}^{-1}$. One advantage of SU8 is a high resistance against acids etc. (e.g. Piranha Solution or plasma etching); for more information see the manufacturers webpage. SU8 has a melting point of $356 \mathrm{~K}$, which defines the maximum for possible temperaturedependent measurements.

With this setup, extremely high shear rates of, for example, $500000 \mathrm{~s}^{-1}$ can be reached with a low amount of sample volume. In our experiments we needed $1.5 \mathrm{ml}$ of sample solution to perform a full experiment consisting of 250 individual measurements. We used a volume speed of $0.1 \mu \mathrm{L} \mathrm{s}^{-1}$, resulting in a shear rate distribution from $0 \mathrm{~s}^{-1}$ up to $300000 \mathrm{~s}^{-1}$. In principle even higher shear rates can be reached by increasing the pumping speed. We note that all these measurements are, despite the high shear rate, carried out far below the transition between laminar and turbulent flow in our small channel.

Due to the small dimension of the channel it was only possible to simulate the shear distribution within the channel. These simulations were carried out for different liquid speeds by the company ASD Advanced Simulation \& Design GmbH, Germany. As input parameter for these simulations the viscosity and volume velocity was given, and finite-element simulations were performed. The viscosity curves were measured with a rotational rheometer, GEMINI 2, HRnano from Malvern, UK. The rheological data are shown in Fig. S1 of the supporting information.

\section{Experiment}

SAXS is a well established technique for investigation of the tertiary structure of proteins and their ordering at high concentrations. The scattered intensity $I(q)$ of a solution of slightly anisotropic or polydisperse particles with strong repulsion (a reasonable approximation for proteins at low concentrations) can be described in a decoupling approximation by (Hayter \& Penfold, 1983, Kotlarchyk \& Chen, 1983)

$$
I(q)=I(0) P(q) S^{\prime}(q)+B,
$$

where $q=(4 \pi / \lambda) \sin \theta$ is the wavevector transfer, $2 \theta$ is the scattering angle and $\lambda$ is the wavelength of the incident photons. Furthermore,

$$
\begin{gathered}
P(q)=\left\langle|F(q)|^{2}\right\rangle, \\
S^{\prime}(q)=1+\beta(q)[S(q)-1], \\
\beta(q)=|\langle F(q)\rangle|^{2} /\left\langle|F(q)|^{2}\right\rangle .
\end{gathered}
$$

The angular brackets $\langle\ldots\rangle$ in equations (2) and (4) represent an average weighted by the distribution of particle sizes and/or orientations, $I(0)$ is the scattering at zero angle which is proportional to the aggregation number of the particles, the concentration of particles and the square of the contrast. $P(q)$ is the form factor, $F(q)$ is the amplitude of the form factor, $S(q)$ is the structure factor, and $S^{\prime}(q)$ is the effective structure factor modified by the anisotropy and/or polydispersity of particles, and $B$ is the residual background.

The measurements were performed at the nanofocus setup of beamline ID13 of the European Synchrotron Radiation Facility (ESRF), Grenoble, France, using an X-ray beam with a size of $250 \mathrm{~nm} \times 250 \mathrm{~nm}$. The photon energy was $13 \mathrm{keV}$ and the detector-to-sample distance was $0.6 \mathrm{~m}$. With these parameters of the setup we were able to access a $q$-range from $0.2 \mathrm{~nm}^{-2}$ up to $2.5 \mathrm{~nm}^{-2}$.

Lysozyme, HEPES and $\mathrm{NaCl}$ were purchased from SigmaAldrich and used without further purification. All lysozyme solutions had a concentration of $50 \mathrm{mg} \mathrm{ml}^{-1}$. We used two buffer compositions, which were either $20 \mathrm{~m} M$ HEPES or $140 \mathrm{~m} M \mathrm{NaCl}$ with $20 \mathrm{~m} M$ HEPES. The $\mathrm{pH}$ value was adjusted to 7 and the temperature of the solution was $293 \mathrm{~K}$. 


\section{Results and discussion}

In the first step we probed the influence of shear on $50 \mathrm{mg} \mathrm{ml}^{-1}$ lysozyme solutions in $20 \mathrm{mM}$ HEPES with $140 \mathrm{mM} \mathrm{NaCl}$ at $\mathrm{pH} 7$ to investigate possible effects of shear forces on the tertiary structure of lysozyme. Fig. 2 shows representative SAXS data obtained at different shear rates. No variation in the scattering curves can be observed. Thus, it can be concluded that shear forces have no effect on the shape of the protein, i.e. it is stable. The shape of the scattering curve at high $q$-values is typical for lysozyme (Grobelny et al., 2014). At low $q$-values an upturn in the slope of the intensity can be seen, indicating protein aggregation. A plot of the data in the Guinier representation $\left\{\ln [I(q)]\right.$ versus $q^{2}$; see Figure $\mathrm{S} 2$ in the supporting information\} shows the presence of two linear regions, indicating objects with two different sizes. From the Guinier approximation the respective radii of gyration can be derived: $(1.3 \pm 0.1) \mathrm{nm}$ and $(3.8 \pm 0.4) \mathrm{nm}$. Lysozyme has been reported to have a radius of gyration of $1.4 \mathrm{~nm}$ (Grobelny et al., 2014; Svergun et al., 1998). Thus, it can be concluded that the small objects are single lysozyme molecules whereas the larger objects with a radius of gyration of $3.8 \mathrm{~nm}$ are most likely aggregates of lysozyme that form due to the high concentration.

The scattering patterns were also checked for an induced anisotropy in the structure induced by the shear field. For this a radial integration was performed and the azimuthal intensity distribution was evaluated. The data are shown in Fig. S3 of the supporting information. No change with shear rate was found, which indicates that the shear field has no effect on the orientation of lysozyme, which is related to its low shape asymmetry (Poon et al., 2000).

In the next step the interaction potential of the lysozyme molecules was no longer screened with an excess of salt and the influence of shear on the lysozyme interaction was studied using $50 \mathrm{mg} \mathrm{ml}^{-1}$ solutions in $20 \mathrm{~m} M$ HEPES buffer only. Again, in the first evaluation step the scattering patterns under these solution conditions were checked for an anisotropy induced by the shear field. No deviation in the scattering patterns was found, indicating no alignment of the proteins even at the highest shear rates (see Fig. S4). Also the data at other positions of the beam (closest $5 \mu \mathrm{m}$ away from the channel wall) were checked and no anisotropy in the scattering pattern was found.

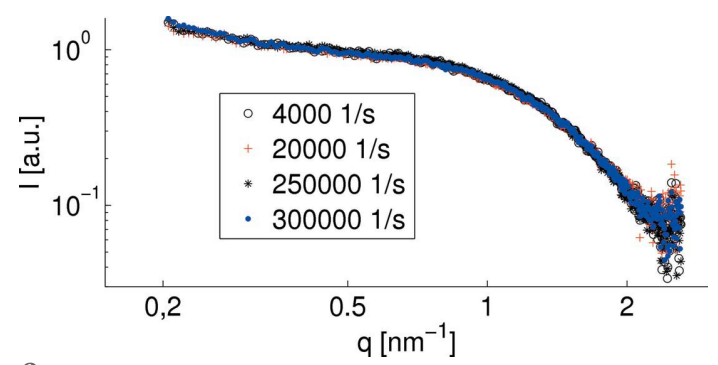

Figure 2

Small-angle scattering data of $50 \mathrm{mg} \mathrm{ml}^{-1}$ lysozyme solutions at different shear rates. The buffer had a $\mathrm{pH}$ of 7 and consisted of $140 \mathrm{mM} \mathrm{NaCl}$ and $20 \mathrm{~m} M$ HEPES.

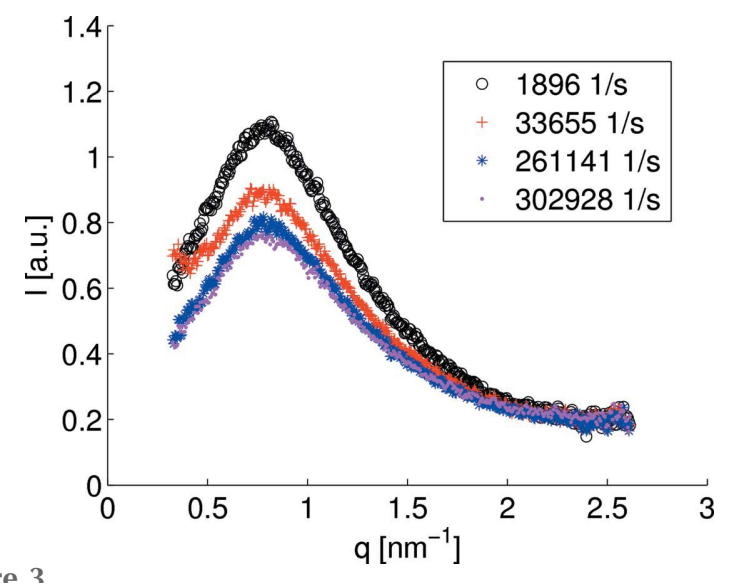

Figure 3

Small-angle scattering data of lysozyme solutions at different shear rates. The concentration was $50 \mathrm{mg} \mathrm{ml}^{-1}$. The buffer had a $\mathrm{pH}$ of 7 and contained $20 \mathrm{~m} M$ HEPES.

Fig. 3 shows selected SAXS patterns from the lysozyme solution obtained at different shear rates. The occurrence of a structure factor, which is indicated by the development of a maximum at $q=(0.8 \pm 0.01) \mathrm{nm}^{-1}$ in the scattering curve, is clearly visible. This can be attributed to increased importance of electrostatic interactions in $20 \mathrm{~m} M$ HEPES, compared with in $20 \mathrm{~m} M$ HEPES with $140 \mathrm{~m} M \mathrm{NaCl}$, due to less screening at the lower ionic strength. Most interestingly, the data show that an increase of the shear rate results in a decrease of the structure factor. For a qualitative analysis the position and amplitude were determined by fitting a Gaussian function to the peak. Fig. 4 shows the evaluated amplitude and position of the maximum in the scattered intensity. The fitted amplitude decreases monotonically with increasing shear rate, but the position of the peak remains constant at $(0.8 \pm 0.01) \mathrm{nm}^{-1}$. From the maximum of the peak an average distance between the scattering objects of $(7.9 \pm 0.8) \mathrm{nm}$ can be estimated (Stradner et al., 2004; Muschol \& Rosenberger, 1997). Our data suggest that this preferred distance between lysozyme molecules is unaffected by shear, but the number of molecules that reside at the preferred distance is reduced at higher shear rates. For charged colloidal system with isotropic distribution the volume fraction of the particles can be deduced from the position of the maximum. The position $q_{\mathrm{c}}$ is connected to the particle number density $n$ by $q_{\mathrm{c}}=2 \pi n^{1 / 3}$, where the particle number density is connected to the volume fraction $\varphi$ by $n=$ $3 \varphi /\left(4 \pi R^{3}\right)$ (Stradner et al., 2004). Calculating the volume fraction by this approach we obtain a value of 0.0425 , which is higher than the volume fraction, 0.033, calculated by the amount of lysozyme dissolved in solution. This indicates that lysozyme molecules in some parts of the solution have a higher particle density compared with if they were homogeneously distributed.

Further analysis has been performed using a model fitting of the scattering intensities. In the modelling we used an ellipsoid of rotation for the form factor $P(q)$ of lysozyme, as previously reported to describe the overall tertiary structure of the protein (Schroer et al., 2011). Further, we assumed that the protein structure was unaltered by shear since no effect of 

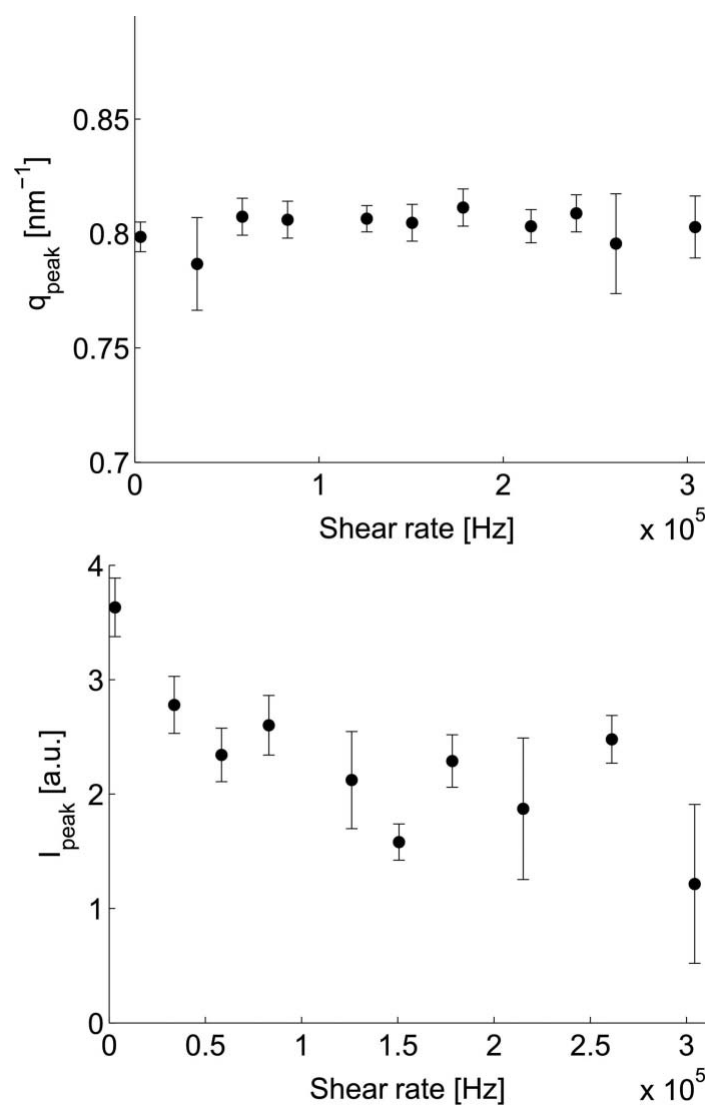

Figure 4

(Top) Peak position as a function of shear rate. (Bottom) Peak intensity as a function of shear rate.

shear forces on the protein shape was observed in the experiment with added $140 \mathrm{~m} M \mathrm{NaCl}$.

Thus, in this model the form factor is given by

$$
P(q)=\int_{0}^{\pi / 2}|F(q, R)|^{2} \sin \beta \mathrm{d} \beta,
$$

where

$$
F(q, R)=3(\sin X-X \cos X) / X^{3}
$$

with

$$
X=q R\left[\sin ^{2} \beta+\gamma^{2} \cos ^{2} \beta\right]^{1 / 2}
$$

and $\gamma=a / b$ describes the ratio of the semi-axis $a$ and $b$ of the ellipsoid of rotation.

For $S(q)$ we used an effective hard-sphere expression as calculated within the Percus-Yevick approximation for the closure relation (Pedersen, 1997, Kinning \& Thomas, 1984),

$$
S(q)=\frac{1}{1+24 \eta_{\mathrm{HS}} G\left(q R_{\mathrm{HS}}\right) / q R_{\mathrm{HS}}}
$$

where $\eta_{\mathrm{HS}}$ is the hard-sphere volume fraction and $R_{\mathrm{HS}}$ is the effective hard-sphere radius. The detailed expression of the function $G\left(q R_{\mathrm{HS}}\right)$, which is the Fourier transformation of the correlation of the positions of lysozyme in solution, can be found in the literature (Pedersen, 1997).
We fitted the form and structure factor in parallel to the data and in total seven fitting parameters have been used: $a, \gamma$, $R_{\mathrm{HS}}, \eta_{\mathrm{HS}}, I(0), R$ and $B$. The parameter $\gamma$ was very close to unity and kept fixed for all further fittings. Reasonable fits have been achieved with an effective hard-sphere interaction. According to the results of the fitting process the form factor suggests that the protein adopts a nearly spherical shape, with a constant radius $R$ of $(1.73 \pm 0.01) \mathrm{nm}$ for the individual lysozyme molecules, and this value is not affected by the shear rate. Thus, the corresponding radius of gyration is $R_{\mathrm{g}}=(1.34 \pm$ $0.01) \mathrm{nm}$. From the fitting of the structure factor a hard-sphere radius $R_{\mathrm{HS}}$ of $3.15 \mathrm{~nm}$ was obtained. This value is lower than the theoretical screening length in a $20 \mathrm{~m} M$ HEPES solution, which according to the Debye-Hückel theory is equal to $5 \mathrm{~nm}$ as the ionic strength of the buffer is $3 \mathrm{mM}$ at $\mathrm{pH}$ 7. The fitted $R_{\mathrm{HS}}$ is higher than the actual radius of the protein, $R$, which is due to the protein charge and the counterions residing next to the protein. We note that the hard-sphere radius is a measure of the minimum distance between proteins, which is increasing with increasing protein charge due to the electrostatic repulsion. The hard-sphere volume fraction obtained from the fitting is 0.15 . The volume fraction depends on the size of the objects, and we therefore have to scale the hard-sphere volume fraction in order to compare with the volume fraction of the protein. This gives an effective volume fraction of 0.052 for the hard-sphere model. This is, again, larger than the volume fraction calculated by the dissolved mass $(\varphi=0.033)$. This indicates that the derived parameters represent the organization of the proteins in ordered configurations where they are closer together and interact strongly due to the electrical charge. We note that the volume fraction calculated from the estimation of the peak position may differ due to the fact that model assumptions have to be made, like a homogeneous distribution of the proteins, a spherical shape and a homogeneous distribution of the charge on the protein, which may affect the obtained results.

For all fitted curves the parameters of the structure factor and form factor remained constant; only the fitting parameter $I(0)$ changed. The decay of $I(0)$ as a function of shear rate is shown in Fig. 5.

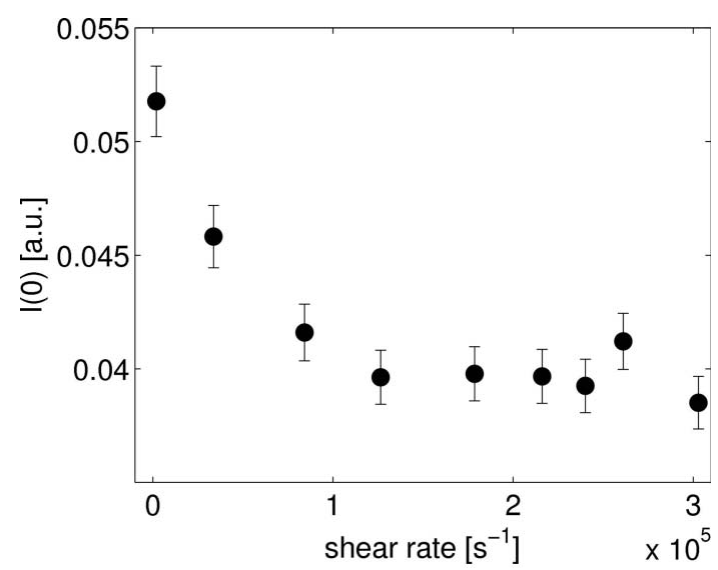

Figure 5

Variation of the fitting parameter $I(0)$ with shear rate. 
The decay is not due to a change of the concentration of the protein, as the concentration of the protein was constant in our experiment. Also, changes induced by radiation damage can be ruled out since our measurements were made on a continuously flowing sample. Furthermore, no change due to shear or changes in the shape of the protein could be detected in lysozyme with added salt. The main factors which are incorporated in the factor $I(0)$ of the modelling is the contrast $(\Delta \rho)^{2}$ and the concentration $n_{\mathrm{C}}$ of the clusters. As there is no argument for a change of the contrast, the only viable explanation is a decrease of the cluster concentration. Thus, the data in Fig. 5 suggest that lysozyme clusters are destabilized by shear forces. A similar effect was also observed by Stradner $e t$ al. (2004) who showed that the structure factor observed in lysozyme solutions decreases with an increase in temperature, which was also explained by dissolution of the equilibrium clusters.

\section{Conclusion}

We have developed a new micro-rheometry setup for in situ $\mathrm{X}$-ray scattering experiments, which allows investigation of structures of and interactions in aqueous solutions (e.g. protein solutions) at high shear rates. In a first experiment the interaction of lysozyme proteins in solution was successfully probed by SAXS. The small and flexible setup is adaptable and can in principle be used at many beamlines provided a sufficiently focused X-ray beam is available.

The measurements showed that single lysozyme proteins are not influenced by the shear forces, and no structural changes could be observed up to $300000 \mathrm{~s}^{-1}$. By using $20 \mathrm{~m} M$ HEPES buffers without any additional salt a structure factor was detected which originates from inter-protein interactions of screened electrostatic nature. The intensity of the structure factor decreases with increasing shear rate. The data analysis suggests that the number of clusters decreases with increasing shear rate, suggesting disintegration of equilibrium clusters of lysozyme by shear forces.

\section{Acknowledgements}

We wish to acknowledge the financial support by the BMBFproject 05K2012 within the Joint International Research Project Röntgen-Angström-Cluster. Further we acknowledge Manfred Burghammer and Britta Weinhausen from ID13, ESRF, France, for support during beam time.

\section{References}

Agarwal, P. K., Somani, R. H., Weng, W. Q., Mehta, A., Yang, L., Ran, S. F., Liu, L. Z. \& Hsiao, B. S. (2003). Macromolecules, 36, 52265235.

Broccio, M., Costa, D., Liu, Y. \& Chen, S. H. (2006). J. Chem. Phys. 124, 084501.

Burghardt, W. R. (1998). Macromol. Chem. Phys. 199, 471-488.

Campbell, A. I., Anderson, V. J., van Duijneveldt, J. S. \& Bartlett, P. (2005). Phys. Rev. Lett. 94, 208301
Cardinaux, F., Zaccarelli, E., Stradner, A., Bucciarelli, S., Farago, B., Egelhaaf, S. U., Sciortino, F. \& Schurtenberger, P. (2011). J. Phys. Chem. B, 115, 7227-7237.

Chen, S.-H., Broccio, M., Liu, Y., Fratini, E. \& Baglioni, P. (2007). J. Appl. Cryst. 40, s321-s326.

Cinader, D. K. \& Burghardt, W. R. (1999). Polymer, 40, 41694180.

Diat, O., Roux, D. \& Nallet, F. (1995). Phys. Rev. E, 51, 32963299.

Dykes, L. M. C., Torkelson, J. M. \& Burghardt, W. R. (2012). Macromolecules, 45, 1622-1630.

Evans, H. M., Dootz, R., Koster, S., Struth, B. \& Pfohl, T. (2007). Bull. Pol. Acad. Sci. Tech. 55, 217-227.

Grobelny, S., Erlkamp, M., Moller, J., Tolan, M. \& Winter, R. (2014). J. Chem. Phys. 141, 22D506.

Groenewold, J. \& Kegel, W. K. (2001). J. Phys. Chem. B, 105, 1170211709.

Hayter, J. B. \& Penfold, J. (1983). Colloid Polym. Sci. 261, 1022-1030.

Hoekstra, H., Mewis, J., Narayanan, T. \& Vermant, J. (2005). Langmuir, 21, 11017-11025.

Hoekstra, H., Vermant, J., Mewis, J. \& Narayanan, T. (2002). Langmuir, 18, 5695-5703.

Hongladarom, K., Ugaz, V. M., Cinader, D. K., Burghardt, W. R., Quintana, J. P., Hsiao, B. S., Dadmun, M. D., Hamilton, W. A. \& Butler, P. D. (1996). Macromolecules, 29, 5346-5355.

Jogun, S. M. \& Zukoski, C. F. (1999). J. Rheol. 43, 847-871.

Kinning, D. J. \& Thomas, E. L. (1984). Macromolecules, 17, 17121718.

Kotlarchyk, M. \& Chen, S. H. (1983). J. Chem. Phys. 79, 24612469.

Kowalczyk, P., Ciach, A., Gauden, P. A. \& Terzyk, A. P. (2011). J. Colloid Interface Sci. 363, 579-584.

Kumaraswamy, G., Issaian, A. M. \& Kornfield, J. A. (1999). Macromolecules, 32, 7537-7547.

Liu, Y., Chen, W. R. \& Chen, S. H. (2005). J. Chem. Phys. 122, 044507.

Liu, Y., Porcar, L., Chen, J. H., Chen, W. R., Falus, P., Faraone, A., Fratini, E., Hong, K. L. \& Baglioni, P. (2011). J. Phys. Chem. B, 115, 7238-7247.

Liu, Y. P., Zhou, W. Q., Cui, K. P., Tian, N., Wang, X., Liu, L. B., Li, L. B. \& Zhou, Y. G. (2011). Rev. Sci. Instrum. 82, 045104.

Lonetti, B., Fratini, E., Casnati, A. \& Baglioni, P. (2004). Colloids Surf. A, 248, 135-143.

Molino, F. R., Berret, J. F., Porte, G., Diat, O. \& Lindner, P. (1998). Eur. Phys. J. B, 3, 59-72.

Muschol, M. \& Rosenberger, F. (1997). J. Chem. Phys. 107, $1953-$ 1962.

Oliveira, M. S. N., Alves, M. A., Pinho, F. T. \& McKinley, G. H. (2007). Exp. Fluids, 43, 437-451.

Panine, P., Narayanan, T., Vermant, J. \& Mewis, J. (2002). Phys. Rev. $E, \mathbf{6 6}, 022401$.

Pedersen, J. S. (1997). Adv. Colloid Interface Sci. 70, 171-210.

Pignon, F., Magnin, A., Piau, J. M., Cabane, B., Lindner, P. \& Diat, O. (1997). Phys. Rev. E, 56, 3281-3289.

Poon, W. C. K., Egelhaaf, S. U., Beales, P. A., Salonen, A. \& Sawyer, L. (2000). J. Phys. Condens. Matter, 12, L569-L574.

Porcar, L., Falus, P., Chen, W. R., Faraone, A., Fratini, E., Hong, K. L., Baglioni, P. \& Liu, Y. (2010). J. Phys. Chem. Lett. 1, 126-129.

Pujari, S., Dougherty, L., Mobuchon, C., Carreau, P. J., Heuzey, M. C. \& Burghardt, W. R. (2011). Rheol. Acta, 50, 3-16.

Rosenbaum, D., Zamora, P. C. \& Zukoski, C. F. (1996). Phys. Rev. Lett. 76, 150-153.

Roux, D. C., Berret, J. F., Porte, G., Peuvrel-Disdier, E. \& Lindner, P. (1995). Macromolecules, 28, 1681-1687.

Schroer, M. A., Markgraf, J., Wieland, D. C. F., Sahle, C. J., Moller, J., Paulus, M., Tolan, M. \& Winter, R. (2011). Phys. Rev. Lett. 106, 178102.

Sciortino, F., Mossa, S., Zaccarelli, E. \& Tartaglia, P. (2004). Phys. Rev. Lett. 93, 055701. 
Sciortino, F., Tartaglia, P. \& Zaccarelli, E. (2005). J. Phys. Chem. B, 109, 21942-21953.

Shukla, A., Mylonas, E., Di Cola, E., Finet, S., Timmins, P., Narayanan, T. \& Svergun, D. I. (2008). Proc. Natl Acad. Sci. USA, 105, 5075-5080.

Somani, R. H., Hsiao, B. S., Nogales, A., Srinivas, S., Tsou, A. H., Sics, I., Balta-Calleja, F. J. \& Ezquerra, T. A. (2000). Macromolecules, 33, 9385-9394.

Stradner, A., Cardinaux, F. \& Schurtenberger, P. (2006). J. Phys. Chem. B, 110, 21222-21231.

Stradner, A., Sedgwick, H., Cardinaux, F., Poon, W. C. K., Egelhaaf, S. U. \& Schurtenberger, P. (2004). Nature (London), 432, 492-495.

Svergun, D. I., Richard, S., Koch, M. H. J., Sayers, Z., Kuprin, S. \& Zaccai, G. (1998). Proc. Natl Acad. Sci. USA, 95, 2267-2272.
Tardieu, A., Le Verge, A., Malfois, M., Bonneté, F., Finet, S., RièsKautt, M. \& Belloni, L. (1999). J. Cryst. Growth, 196, 193-203.

Toledano, J. C. F., Sciortino, F. \& Zaccarelli, E. (2009). Soft Matter, 5, 2390-2398.

Trebbin, M., Steinhauser, D., Perlich, J., Buffet, A., Roth, S. V., Zimmermann, W., Thiele, J. \& Forster, S. (2013). Proc. Natl Acad. Sci. USA, 110, 6706-6711.

Versmold, H., Musa, S., Dux, C., Lindner, P. \& Urban, V. (2001). Langmuir, 17, 6812-6815.

With, S., Trebbin, M., Bartz, C. B. A., Neuber, C., Dulle, M., Yu, S., Roth, S. V., Schmidt, H. W. \& Förster, S. (2014). Langmuir, 30, 12494-12502.

Wu, J. L., Liu, Y., Chen, W. R., Cao, J. S. \& Chen, S. H. (2004). Phys. Rev. E, 70, 050401. 